\title{
Designing a Gamified Adherence System for Tuberculosis Treatment Support in Urban Vietnam
}

\author{
Nadine Kathrin Ostern \\ Frankfurt School of Finance and \\ Management \\ $\underline{\text { n.ostern@fs.de }}$
}

\author{
Guido Perscheid \\ Frankfurt School of Finance and \\ Management \\ g.perscheid@fs.de
}

\author{
Jürgen Moormann \\ Frankfurt School of Finance and \\ Management \\ j.moormann@fs.de
}

\begin{abstract}
Tuberculosis remains one of the deadliest infectious diseases in Vietnam. Its occurrence is exacerbated by erratic treatment, in particular by taking medication only sporadically or if patients discontinue the medication in an early stage. Previous approaches to support treatment adherence focus on monitoring (e.g., tele-observation) or external stimulation (e.g., rewards). These approaches, however, have not yet shown the desired effects. We assert that this is because current approaches focus on combatting the outcome, i.e., erratic treatment, instead of tackling the reasons for treatment noncompliance. Notably, the latter is heavily related to the stigmatization of TB patients and, especially, selfstigmatization. Using a design science research approach, this paper proposes a research plan for developing a gamified information system that aims at reducing patients self-stigmatization, by providing features that support TB patients community building as well as TB patients empowerment.
\end{abstract}

\section{Introduction}

Being one of the top ten causes of death globally as well as the leading cause of death from a single infectious agent ranking even above HIV, tuberculosis (TB) remains an urgent public health threat $[1,2]$. While a timely diagnosis and treatment with first-line antibiotics for six months can cure most patients and prevents onward transmission, erratic treatment adherence leads to the continued spread of the infection, disease chronicity, and especially the acquisition of multi-drug resistant forms of TB (MDRTB) [3]. In a recent report, the World Health Organization (WHO) draws attention to about half a million new cases of MDR-TB globally in 2018, whose treatment with second-line antibiotics is significantly longer (from 9 up to 20 months) and more expensive ( $\geq$ U.S.\$ 1,000 per person) than the treatment of non-resistant forms of the infection [4].
At the same time, the treatment success rate of MDRTB is only at $56 \%$ globally [1].

As MDR-TB provides a severe threat to the WHO's efforts aiming at containing TB infections, several studies focus on the circumstance of patients developing drug-resistant forms of TB. Qualitative studies suggest that interrupted treatment and missing drug doses is a significant risk factor for developing MDR-TB, whereas one crucial determinant of treatment non-compliance is TB patients' stigmatization $[5,6]$.

Stigma involves the exclusion, rejection, blame or devaluation resulting from experience or reasonable anticipation of an adverse social judgment [7], thereby being a social determinant that affects health outside of the ease with which an individual can access medical services [6]. Notably, there are several reported cases where TB stigma harmed treatment adherence and the regular intake of drug doses. For instance, TB stigma was the most common motivation cited by HIV-infected Tanzanian patients who did not complete isoniazid preventive therapy [8]. Also, TB stigma is associated with non-compliance among Pakistani TB patients on direct observatory therapy, i.e., a specific strategy of the WHO to improve adherence by requiring health workers, community volunteers, or family members to observe patients in being compliant with their prescribed medical treatment $[6,9]$.

Given the stigmatization of TB patients and the associatively increased likelihood of developing MRD-TB through a discontinued use of medication, researchers aim at supporting treatment adherence using new information and communication technologies (ICTs), especially, by developing digital health services. Digital health services are seen as especially promising as they provide novel opportunities to combat TB. Thus us because, as global connectivity expands and modern ICTs become more widely available and affordable, digital health 
services are destined to become increasingly present ans supportive in the daily life of TB patients [2,3].

So far, however, studies investigating the effectiveness of digital or mobile health services on patients health and treatment adherence attested only limited impact. For instance, interactive SMS reminders [10], as well as direct video observation used to control drug intake, showed only limited effectiveness [11,12]. Researchers have shown that SMS reminders and direct video observation instead serve as a reminder for patients who have demonstrated prior good adherence, but that they are not effective if in the case of patients making an active effort to avoid therapy, e.g., pretending to swallow.

This paper argues that the reasons for the current lack of efficiency of digital health services aiming at fostering TB treatment adherence might be two folded. First, current health services focus on the outcome and control aspects of TB treatment adherence rather than on the causes of treatment non-compliance, which is frequently observed to be TB stigma as well as consequential effects, such as social isolation, the loss of job and working hours, and food insecurity. Second, by focusing on the outcome instead of the causes of treatment non-compliance, patients are exposed to high external pressure, eventually leading to a lower individual sense of self-efficacy and self-control $[3,13,14]$.

Therefore, we propose a digital health service that fosters personal responsibility for one's own health on the one side, while helping patients to cope with TB stigma on the other side. We intend to accomplish this by assisting patients to build communities among infected, thereby integrating gamified design elements that foster personal engagement, a sense of belonging, and digital interaction in the course of the TB treatment. Specifically, we focus on the aspect of community building, since it has already been shown to be an effective tool against the spread of $\mathrm{TB}$ and HIV/AIDS in Africa, achieved through the empowerment of patients and the increase of selfresponsibility [6].

Putting these insights together, this paper is dedicated to describe a comprehensive research plan to design and develop a gamified TB treatment adherence system that not only tackles the issue of treatment non-compliance but also fosters patients' engagement in TB therapy. Drawing on cognitive evaluation theory, we argue that the current health services are rarely sufficient to keep patients engagement in their long-term treatment plan, as they are solely exposed to extrinsic motivation (i.e., the use of controls or reward programs). By designing a gamified system, however, we focus on fostering intrinsic motivation, which is consistently more predictive of human behavior than extrinsic motivation $[15,16]$.

Although often reduced to joy alone, intrinsic motivation involves other factors such as the need for competence (i.e., self-esteem) and autonomy (i.e., personal control), whose activation enables patients to take more responsibility for their health [17]. Aimed at triggering intrinsic motivation, we implement game design elements (e.g., collecting badges or reward points, visualization of achievements, social discovery, or quests) that foster community building and exchange between those affected. As a result, we expect patients to empower themselves and others, which actively reduces the negative effects of $\mathrm{TB}$ stigmatization.

The remainder of this paper is structured as follows: The next section briefly describes the framework that guides our approach in designing a gamified system for TB treatment adherence. Notably, we elaborate on the elements of the framework, including our target system, i.e., a district in Ho-ChiMinh city in Vietnam, which is known to be a highburden MDR-TB country [1]. In the following section, we propose research steps needed to be conducted to design a gamified system for TB drug adherence as outlined above. As we apply design science research, we further explain and specify the assessment criteria for the system and eventually provide a conclusion and outlook.

\section{Gamified System Design Framework}

Gamification is defined as the incorporation of game design elements into a target system [18]. While often confused with full-fledged games, gamification merely adds a layer to the real world, without scarifying real-world functionalities. The design of a gamified system, thus, is imbued with high complexity since there is the need for taking into account both real words and game functionalities. Given this complexity, [18] developed a framework that guides researchers in their attempt to design gamified systems, as depicted in Figure 1. Notably, the framework is divided into four building blocks, which we describe briefly in the following.

\subsection{Gamified System}

The first building block is the gamified system, which, consisting of both gamification objects and gamification mechanics. Gamification objects thereby describe the basic building blocks of a gamified system, including items, characters, or visual assets, which either serve to create sensory experiences or provide functionalities. Gamification mechanisms 
refer to the rules that govern the interaction between the user and the game objects, e.g., rules concerning how many points users get for a specific activity in a reward-based game [18]. Notably, several possible gamification mechanics mainly depend on the target systems' characteristics, meaning that design elements need to be congruent with the targeted users, tasks, and the technology [18].
Gamified principles, as well as all other building blocks of the gamified system, however, need to be carefully planned, reviewed constantly, and be discarded if necessary. This is because gamified design systems often do not work as intended as divergent dynamics emerge while in use. Particularly, this means that there is a paucity of practice-based wisdom that can guide and promise a successful

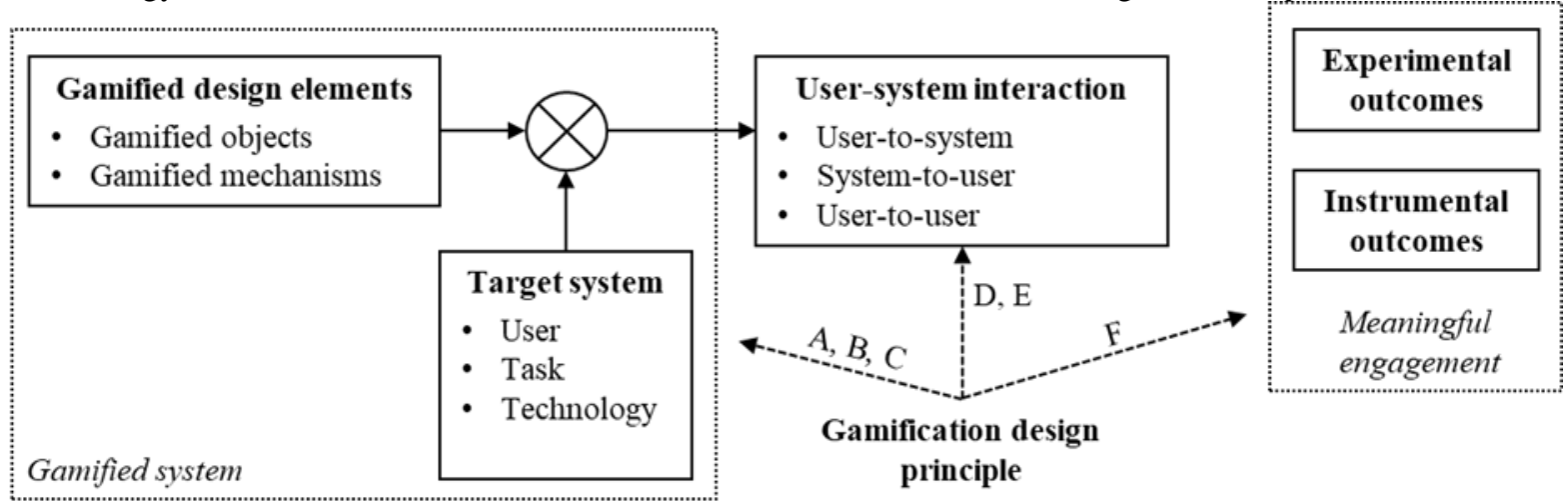

Figure 1. Framework to design a gamified system

\subsection{User-System Interaction}

Gamification mechanics plus actions taken by the user result in user-system interaction, which is the second building block of the gamified system design framework. While the importance of user-system interaction is often underestimated in design science research approaches (as they are typically solutionoriented) [19], in gamified systems, user-system interaction is of utmost importance and includes system-user communication as well as communication with other users [18]. Eventually, setting up the usersystem communication significantly influences the quality of the communication, e.g., how feedback is present to users and determines its overall acceptance among users.

\subsection{Gamification Design Principles}

Gamification design principles or higher-level design principles are formulas or rules for designers. For instance, design principles can be expressed in how users onboard to the game, by keeping outcomes uncertain or providing frequent rewards and immediate feedback supporting different user styles [18]. Examples of gamification design principles include the use of timely feedback, the ability to set personalized goals and messages as well as social supports, e.g., to give other users positive encouragement [18]. development and implementation of our intended

gamified system. Consequently, when elaborating on our intended outcome, i.e., meaningful engagement, we need to obey that designing for this outcome is probably hard as it emerges over time and patients' interest to work and engage within the system [18].

\subsection{Meaningful Engagement}

Eventually, meaningful engagement represents the overarching goal of a gamified system. In particular, [18] describe meaningful engagement to be consisting of two building blocks, i.e., instrumental and experimental outcomes. While instrumental outcomes are purpose orientated, i.e., the increase of sales or inclines in the total number of users, experimental outcomes address the psychological state of a recipient's perception by, for example, creating positive feelings or emotions that lead to an improved engagement with the gamified system. Experimental outcomes should, thereby, not be understood as a mere additional benefit in the sense of positive side effects, but as equally important as the instrumental outcomes [18]. Notably, [18] recommend the identification of desired experimental outcomes before the gamified system is designed in order to support the creation of instrumental outcomes. 


\section{Research Plan: Toward Designing a Gamified System for TB Drug Adherence}

We follow a design science research (DSR) approach [19] to develop and design a gamified drug adherence system that incorporates and obeys the building blocks of the above-presented framework [18]. Thereby, we build upon accumulated design knowledge in the solution space, i.e., we use the framework that was built to solve related problems (i.e., its usage to design gamified systems) and transfer this knowledge to the contextual problem space of TB stigmata and its relationship with TB treatment adherence in urban Vietnam [20].

We strive for a mainly solution-oriented DSR project by going through the six main phases of the DSR process, i.e., problem identification and motivation, definition of objects, design and development, demonstration, evaluation, and communication [19,21]. To achieve patients' engagement in community building and to foster TB patients' empowerment, we use the described framework [18], concerned with the design of gamified systems for guidance, and as a knowledge base to develop and design the treatment adherence system.

We start describing our approach by opening up the problem space and describing the target system in the following.

Problem space and target system: The DSR approach, as well as the framework, require - in a first step - to fully grasp the problem space residing within the target system. We aim at implementing a gamified TB adherence system in a district of Ho-Chi-Minh City, Vietnam, which is known to be profoundly affected by TB [1]. Vietnam is a middle-income country in Southeast Asia, with a TB incidence of 182 per 100,000 population in 2018 [1]. While the country makes ongoing progress in reducing the overall $\mathrm{TB}$ burden by approximately $3 \%$ per year over the past ten years through a range of interventions (e.g., household contact investigation, TB preventive treatment, new TB diagnostics, active case finding), the WHO classifies Vietnam as a high TB and, especially, high MDR-TB burden country [4]. Tuberculosis control is centrally administered by the National TB Program (NTP), and first-line medications are dispensed freeof-charge, mostn commonly once a week by health workers at district clinics or commune health posts [22]. Despite the free dispense of antibiotics and support from various NGOs, TB remains one of the most severe forms of infectious diseases in Vietnam, which is, among others, because of the still predominant stigmatization of TB patients, whereby stigmatization is a social determinant of health [6]. In particular, it has been shown that the prevalence of the $\mathrm{TB}$ in Vietnam is even mainly due to social determinants and indirect costs of the disease, including, among others, stigmatization, loss of employment, discrimination, and additional costs due to extra travel or food supplementary costs [6].

Design objectives: Following the DSR methodology, we aim to define objectives, i.e., what a (better) artifact has to accomplish, in a second step [19]. We, thereby, target each of the building blocks of the gamified design framework subsequently, starting with the outcome, i.e., the instrumental and experimental outcome which comprise meaningful engagement, as proposed by [18]. Table 1 summarizes the key guiding research questions, the proposed approach, as well as the objective for each of the framework's building blocks. Within the framework of the design project, we will work through these steps one after the other and divide them into individual research projects.

Demonstration: DSR research requires the demonstration of the artifact, e.g., the prototype designed. While this is out of the scope for this paper, it is intended to be addressed in future works after all the design-specific characteristics of the system have been specified. Notably, this also holds for the communication of results, being the final step of the DSR methodology [19]

Table 1. Framework building blocks and proposed research steps

\begin{tabular}{|l|l|l|l|}
\hline $\begin{array}{l}\text { Framework } \\
\text { Building } \\
\text { Blocks }\end{array}$ & Key guiding research question & Research Approach & Design Objectives \\
\hline $\begin{array}{l}\text { Step 1: } \\
\text { Meaningful } \\
\text { engagement }\end{array}$ & $\begin{array}{l}\text { What is the patients' desired } \\
\text { instrumental and experimental } \\
\text { outcome when using the gamified } \\
\text { system? }\end{array}$ & $\begin{array}{l}\text { Identification of issues and factors } \\
\text { that prevent the continuous intake of } \\
\text { TB medication using semi-structured } \\
\text { interviews [23] }\end{array}$ & $\begin{array}{l}\text { Create a system design that } \\
\text { incorporates functionalities } \\
\text { required by TB patient, which } \\
\text { enhance the feeling of control } \\
\text { and empowerment from a ' } \\
\text { patient's view }\end{array}$ \\
\hline $\begin{array}{l}\text { Step 2: } \\
\text { Gamified } \\
\text { System }\end{array}$ & $\begin{array}{l}\text { What are the concrete game } \\
\text { objects and mechanics that } \\
\text { promote meaningful engagement }\end{array}$ & $\begin{array}{l}\bullet \text { Literature overview of already } \\
\text { applied gamified systems, the } \\
\text { context, and their effectiveness. }\end{array}$ & $\begin{array}{l}\text { Identification and } \\
\text { implementation of concrete } \\
\text { design elements that promote }\end{array}$ \\
\hline
\end{tabular}




\begin{tabular}{|c|c|c|c|}
\hline & & $\begin{array}{l}\text { - Creation of an evaluation matrix that } \\
\text { helps to classify the usefulness of } \\
\text { gamified elements from a 'user's } \\
\text { perspective or rating [24] }\end{array}$ & $\begin{array}{l}\text { the instrumental and } \\
\text { experimental outcomes from } \\
\text { the user's perspective }\end{array}$ \\
\hline $\begin{array}{l}\text { Step 3: } \\
\text { Gamification } \\
\text { principles }\end{array}$ & $\begin{array}{l}\text { How can we translate the relation } \\
\text { of patients' needs and game } \\
\text { objects/mechanics into higher- } \\
\text { order principles, i.e., core } \\
\text { properties of the gamified } \\
\text { system? }\end{array}$ & $\begin{array}{l}\text { - Literature review on gamification } \\
\text { principles (e.g., autonomy, local } \\
\text { interactivity, rules of engagement, } \\
\text { communication) and their } \\
\text { effectiveness [25] } \\
\text { - Mapping of identified mechanism to } \\
\text { the target system and game objects/ } \\
\text { mechanics [26] }\end{array}$ & $\begin{array}{l}\text { Identification and } \\
\text { actualization of affordances of } \\
\text { the gamified system by } \\
\text { design, i.e., providing general } \\
\text { action possibilities opened up } \\
\text { by the system to the patient. }\end{array}$ \\
\hline $\begin{array}{l}\text { Step 4: } \\
\text { User-system } \\
\text { interaction }\end{array}$ & $\begin{array}{l}\text { What are the desired } \\
\text { technological and functional } \\
\text { affordances of the gamified drug } \\
\text { adherence system? } \\
\text { How can we design a gamified } \\
\text { system such that affordances are } \\
\text { immediately perceptible by users? }\end{array}$ & $\begin{array}{l}\text { - User observation of patients using a } \\
\text { prototype of the gamified system } \\
\text { [27] } \\
\text { - Adaption of the prototype to design } \\
\text { for affordance actualization [28] }\end{array}$ & $\begin{array}{l}\text { Identification and } \\
\text { actualization of affordances of } \\
\text { the gamified system by } \\
\text { design, i.e., providing general } \\
\text { action possibilities opened up } \\
\text { by the system to the patient. }\end{array}$ \\
\hline
\end{tabular}

While there is no prototype readily available now, we want to use this paper to demonstrate the expected and intended impacts of the system on the current state and relationship between the government, NGOs, public and private healthcare facilities, and patients. Figure 2 shows these relationships and associated responsibilities characterizing the state of control of and care for TB and TB patients in Vietnam.

Following [8] and [13], the current TB control and care system creates barriers to patients treatment success and adherence that are, among others, caused by the distance of patient's houses to clinics they have to visit in order to receive their treatment as well as the time and costs for traveling to private or public clinics.

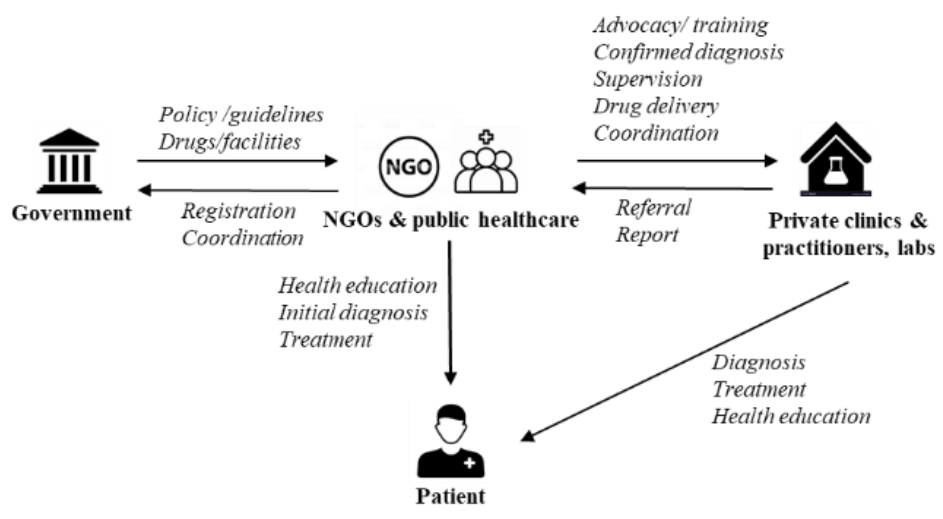

Figure 2. Current TB control and care

Moreover, patients recorded that they often forgot or intentionally omitted scheduled appointments due to expected stigmatization, meaning that both practical and psychosocial barriers to attend and to adhere to treatments are present in the current TB control and care system [29].

While it would be naïve to assume that scholarly recommendations and proposed interventions aimed at improving practical aspects and the functioning of the TB control and care system, are capable of sustainably changing the TB control and care system, instead, we propose evoluationary rather than disruptive measures to improve TB control and care in Vietnam. Notably, we want to demonstrate how the envisaged gamified system is likely to be integrated into the existing system thereby expected to decrease psychosocial barriers for patients. Figure 3 exemplary depicts the integration of the gamified system in the current TB control and care system. With the introduction of the envisaged gamified system, we intend to stimulate patients' engagement. However, we also expect several cross effects to emerge that enhance and make the existing TB control and care system more efficient. Notably, we expect distinguishing impacts of the gamified system to unfold on three levels. Thereby, we expect distinguishing impacts of the gamified system to unfold on an administrational, informational, and a personal level. 


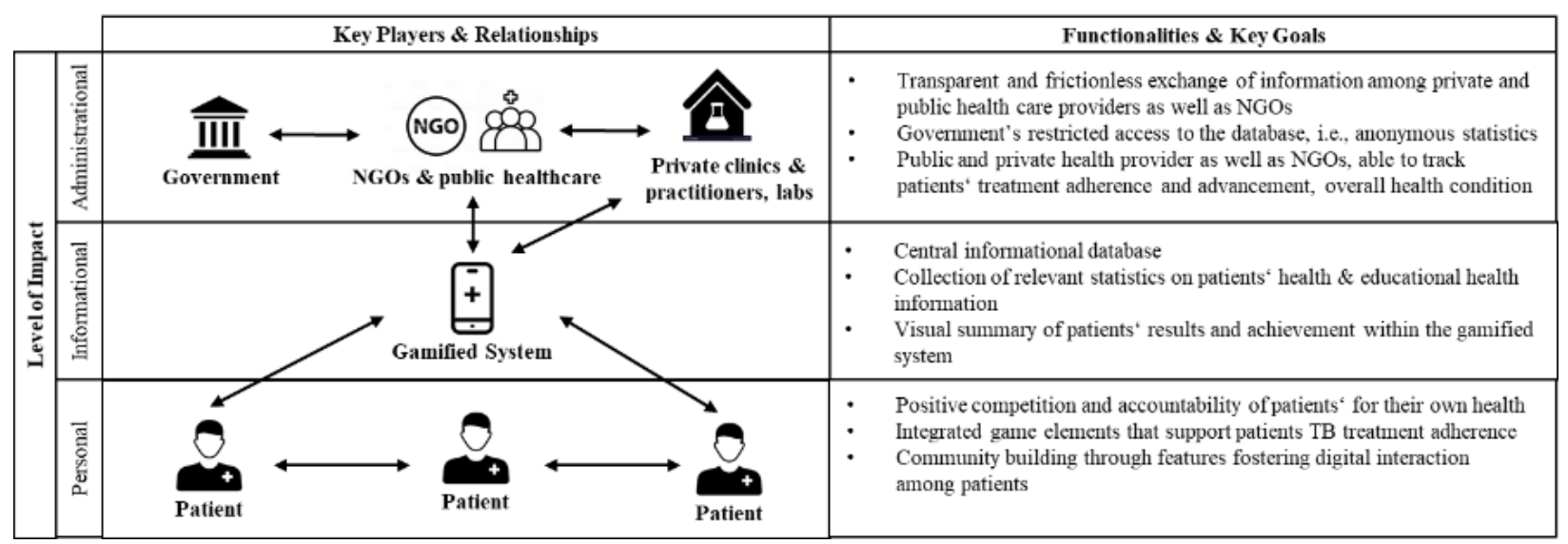

Figure 3. Expected impact of the gamified system on TB control and care

First, the envisaged gamified systems is intended to increase patients' engagement in well as acountability for patients' own health. Thereby, we expect an impact on the patients' personal level, which we define as patients' level of engagement in their TB treatment, arising from the use of the system for achieving and mastering game elements and mechanisms. The gamified systems also enables patients to compare their achievements with the ones of others, cheer each other up, as well as to get in touch with other patients. These features are enabled by the gamified systems' core functionalities, as summarized in the figure.

Second, by using the system and engaging in gamified elements, health care service providers might receive insights into patients' overall health education, knowledge on TB and its dissemination. Vice versa, health care service providers are able to upload educational health information on the system, as well as potentially creating gamified challenges on their own by which means they were able to obtain further patient-related user data, enabling the analysis and evaluation of a patients individual health status. Reminder functions, e.g., for appointments or drug intake, can be easily integrated into the system through the health service provider, which positively affects the administrational level of the TB control and care system. We define impacts of the administrational level as the extent of administrational ease that is achieved by the voluntary completion of playful tasks by patients and the release of data, which enables appointment reminders and other automated functionalities.

Eventually, on an informational level, the gamified system serves as a centralized database that captures educational health information, patients' health status as well as additional information, such as weight and age, given the prerequisite that patients are willing to share these informations. We define the impact on the informational level as the degree of integration and share-ability of information through

the digital storage of health-related data via the gamified system.

Evaluation: For the evaluation of the envisaged gamified TB drug adherence systems, we will assess the following evaluation criteria, i.e., feasibility, usability, and acceptability. While we will attach great importance to the rigor of the research projects in the concrete development of the prototype, these criteria are initially aimed to show the applicability of the gamified system, since the overall success, i.e., the real-world impact, will be measured by this [30].

Feasibility: Following [31], we define feasibility in the context of the gamified system as the extent to which we expect the implementation of the gamified system to be "easy" and convenient. Notably, we will account for how "easy" the system is integrable into the daily health routine of TB patients, as well as whether the system introduced inconvenience to patients or governmental or non-profit health care providers. Furthermore, we will assess whether the patients' mental load during thesuse of the system is manageable and whether if staff is needed to train patients to integrating the app into their daily life and health care routines [31].

While these factors need to be tested, we expect mixed outcomes to result from the feasibility test. This is because Vietnam is among the countries with the highest rate of mobile phone ownership (131 mobile phone subscriptions per 100 inhabitants), and approximately 38,4 million are smartphone users [32]. Consequently, we expect that less effort will have to be invested into the integration of the system regarding patients' daily life if we provide mobile accessibility to the gamified system. At the same time, however, integration might be tricky if patients refuse to take their daily medication, either because they do not 
believe in the treatment or try to cover up the infection $[6,33]$.

Usability: Usability is defined as the extent to which patients can adequately record and track data concerning their treatment adherence and whether or not the functions of the gamified systems enhance treatment compliance [31]. We, therefore, aim to test the effectiveness of the system in terms of both treatment compliance, as well as stigma reduction. While the first can be easily tracked using the system data, as well as health data, being easily integrated into the gamified system by providing interfaces to general health practitioners or hospitals treating TB patients, we will assess stigma reduction, using a stigma assessment scale [34,35]. In particular, we will develop and test a self-stigma scale to measure stigma patients suspected to have TB. In particular, we focus on self-stigmatization as we assume a lower level of self-stigmatization to be equal with patients' empowerment. This is because, while it is tough to change social believes about a disease within the society [6], our approach strives to changes the perception of stigmatization by patients through community-building and empowerment by the means of the gamified system.

Acceptability: Acceptability of the gamified systems refers to question whether or not the gamified system is likable by TB patients, including the interface and navigation features [31]. To ensure acceptability, we will conduct several iterations of preprototype- and prototype acceptance testing [36]. Therefore, we are optimistic about designing a gamified TB treatment adherence system that is useful and acceptable to TB patients. However, there are overarching issues that affect usability and, especially, the acceptability of the gamified system, which require special investigation and testing.

Overarching considerations: System and, especially, interface design are driven by cultural aspect [37]. Thus, we need to take into account that the initial design of the interface needs to be revised by several iterations until we reach a good culturetechnology fit. Otherwise, our system runs the risk of ,despite providing suitable features, not being accepted and, consequently, being effective [38]. Besides cultural aspects, differences in gender are also prevalent and should be taken into consideration. For instance, it has been shown that female TB patients have stronger fears about the consequences of their illness, including the misbelief that $\mathrm{TB}$ medication leads to sterility [39]. Therefore, we expect more resistance among female TB patients to test and use the gamified system, which we need to consider when designing the TB drug adherence system, e.g., by offering an incognito mode to test the gamified system.

\section{Conclusion and Outlook}

This paper provides initial insights into a comprehensive research project that aims to introduce a gamified system to support tuberculosis (TB) drug adherence by fostering engagement of patients in the course of their treatment through community-building and TB patients' empowerment. We propose to address the problem at the root by empowering TB patients through community building that is fostered by game design elements. In this research-in-progress paper, we focus on the objectives and evaluation criteria of our solution, which are subject to identification and investigation by empirical research. The relevance of this project is evident, when looking at the ever-present amount of multi-drug resistant cases of TB in Vietnam, that exist despite the public accessibility and free provision of TB tests and antibiotics through the Vietnamese government. Additionally, numerous non-governmental institutions advise and support patients with respect to the continuity of drug intake, thereby offering social care and health care.

Moreover, while several solutions exist that already integrate new information and communication technologies in the treatment surveillance, they do not show the desired effectiveness. We submit that this is because they focus on the outcome, i.e., treatment noncompliance, instead of focusing on the reasons for non-compliance. Among others, TB stigmatization is one such reason for treatment non-compliance.

The proposed research project and approach to tackling TB treatment non-compliance in Vietnam, of course, needs to be viewed in light of the limitations. Primarily this means that we present a research project being in a very early stage of its development. However, given the social and practical relevance, as well as the theoretical foundation on which this proposal builds, we are convinced that this paper is of relevance for practitioners and researchers, encouraging discussions on the proposed solution, as well as on the use of gamified systems to address healthcare issues more generally.

\section{References}

[1] WHO, Country Profiles for 30 High TB Burden Countries, St. Gallen, Switzerland, 2019.

[2] WHO, Digital Health for the End TB Strategy: Developing Priority Products and Making Them Work, Geneva, Switzerland, 2016.

[3] S.A. Munro, S.A. Lewin, H.J. Smith, M.E. Engel, 
A. Fretheim, J. Volmink, PLOS Med. 4 (2007) 1230-1245.

[4] WHO, Global Tuberculosis Report 2019, Geneva, Switzerland, 2019.

[5] A.M. Kipp, P. Pungrassami, P.W. Stewart, V. Chongsuvivatwong, R.P. Strauss, A. Van Rie, Int. J. Tuberc. Lung Desease 15 (2011) 1540-1545.

[6] A. Courtwrith, A.N. Turner, Public Health Rep. 125 (2010) 34-42.

[7] M.G. Weiss, J. Ramakrishna, Lancet 367 (2006) 536-538.

[8] P.J. Munseri, E.A. Talbot, L. Mtei, C. Fordham von Reyn, Int. J. Tuberc. Lung Desease 12 (2008) 1037-1041.

[9] J. Karumbi, P. Garner, Cochrane Database Syst. 5 (2015) 1-57.

[10] M.D. Nglazi, L.G. Bekker, R. Wood, G.D. Hussey, C.S. Wiysonge, BMC Infect. Dis. 13 (2013) 1-16.

[11] J. DeMaio, L. Schwartz, P. Cooley, A. Tice, Clin. Infect. Dis. 33 (2001) 2082-2084.

[12] R.S. Garfein, K. Collins, F. Munoz, K. Moser, P. Cerecer-Callu, F. Raab, P. Rios, A. Flick, M.L. Zuniga, J. Cuevas-Mota, K. Liang, G. Rangel, J.L. Burgos, T.C. Rodwell, K. Patrick, Int. J. Tuberc. Lung Desease 19 (2015) 1057-1064.

[13] C. Laxmeshwar, A.G. Steward, A. Dalal, A.M. V. Kumar, S. Kalaiselvi, M. Das, N. Gawde, S.S. Thi, P. Isaakidis, Int. J. Tuberc. Lung Desease 23 (2019) 73-81.

[14] T. Shimamura, A. Taguchi, S. Kobayashi, S. Nagata, J.K. Magilvy, S. Muashima, Public Health Nurs. 30 (2012) 370-378.

[15] E. Deci, R.M. Ryan, in: M. Kemis (Ed.), Effic. Agency, Self-Esteem, Plenum, New York, NY, USA, 1995, pp. 31-49.

[16] P.B. Lowry, B. Hammer, J.E. Gaskin, T.L. Roberts, N.W. Twyman, J. Assoc. Inf. Syst. 14 (2013) 617-671.

[17] J. Macq, T. Torfoss, H. Getahun, Trop. Med. Int. Heal. 12 (2007) 873-885.

[18] D. Liu, R. Santhanam, J. Webster, MIS Q. 41 (2017) 1011-1034.

[19] K. Peffers, T. Tuunanen, M.A. Rothenberger, S. Chatterjee, J. Manag. Inf. Syst. 24 (2008) 45-78.

[20] J. vom Brocke, R. Winter, A.R. Hevner, A. Maedche, J. Assoc. Inf. Syst. 21 (2020) 520-544.

[21] J. Venable, J. Pries-Heje, R. Baskerville, in: K. Peffer, M. Rothenberge, B. Kuechler (Eds.), Des. Sci. Res. Inf. Syst. Adv. Theory Pract., Springer, Heidelberg, 2012, pp. 423-438.

[22] T.A. Nguyen, M.T. Pham, T.L. Nguyen, V.N. Nguyen, D.C. Pham, B.H. Nguyen, G.J. Fox, Int. J. Infect. Dis. 65 (2017) 85-89.

[23] U. Schultze, M. Avital, Inf. Organ. 21 (2011) 116.

[24] N. Melville, K. Kraemer, V. Gurbaxani, MIS Q. 28 (2004) 283-322.

[25] B. Morschheuser, L. Hassan, K. Werder, J. Hamari, Inf. Softw. Technol. 95 (2018) 219-237.

[26] A.R. Chaturvedi, D.R. Dolk, P.L. Drnevich, MIS
Q. 35 (2011) 673-684.

[27] Y.S. Kim, M.K. Kim, J. Jeong, J.A. Park, in: Int. Conf. Eng. Des., Stanford, CA, USA, 2009, pp. 112.

[28] E. Benbunan-Fich, Eur. J. Inf. Syst. 28 (2019) 256-271.

[29] G.J. Fox, L.P. Loan, N.V. Nhung, N.T. Loi, D.N. Sy, W.J. Britton, G.B. Marks, BMC Infect. Dis. (2015).

[30] M. Rosemann, I. Vessey, MIS Q. 32 (2008) 1-22.

[31] J.D. Rothstein, L. Jennings, A. Moorthy, F. Yang, L. Gee, K. Romano, D. Hutchful, A.B. Labrique, A.E. LeFevre, Int. J. Telemed. Appl. (2016) 1-14. [32] Statista, Digit. Mark. Outlook (2017).

[33] N. V. Nhung, N.B. Hoa, N.T. Anh, L.T. Ngoc Anh, A. Siroka, K. Lönnroth, G.I. Baena, Int. J. Tuberc. Lung Desease 22 (2018) 983-990.

[34] G. Rafal, A. Gatto, R. DeBate, J. Am. Coll. Heal. 66 (2018) 284-291.

[35] D.L. Vogel, N.G. Wade, S. Haake, J. Couns. Psychol. 53 (2006) 325-337.

[36] F.D. Davis, V. Venkatesh, IEEE Trans. Eng. Manag. 51 (2004) 31-46.

[37] J. Iivari, M. Huisman, MIS Q. 31 (2007) 35-58.

[38] I. Lee, J. Kim, S.J. Hong, Int. J. Electron. Commer. 11 (2007) 11-51.

[39] N.H. Long, E. Johansson, V.K. Diwan, A. Winkvist, Health Policy (New. York). 58 (2001) 69-81. 